\title{
Tomography reconstruction using the Learn and Apply algorithm
}

\author{
F. Vidal ${ }^{1, \text { a }}$, E. Gendron ${ }^{1}$, M. Brangier ${ }^{1}$, A. Sevin ${ }^{1}$, G. Rousset ${ }^{1}$, and Z. Hubert ${ }^{1}$ \\ Laboratoire d'Etudes Spatiales et d'Instrumentation en Astrophysique (LESIA), Observatoire de Paris- \\ Meudon, CNRS, UPMC, Université Paris Diderot, 5 Place Jules Janssen, 92190 Meudon, France
}

\begin{abstract}
In the framework of the MOAO demonstrator CANARY, we developped a new concept of tomography algorithm that allows to measure the tomographic reconstructor directly on-sky, using or not, a priori from the turbulence profile. This simple algorithm, working in open-loop, uses the measured covariance of slopes between all the wavefront sensors (WFS) to deduce the geometric and atmospheric parameters that are used to compute the tomographic reconstructor. This method called "Learn and Apply" (L\&A) has also the advantage to measure and take into account all the misalignments between the WFSs in order to calibrate any MOAO instrument. We present the main principle of the algorithm and the last experimental results performed in MOAO scheme at the SESAME bench.
\end{abstract}

\section{Context}

One of the key scientific drivers of the future giant telescopes of the class $30-50 \mathrm{~m}$ is the formation of the early universe, and in particular the way distant galaxies assembled. These extremely dim and small objects require both the large light-collecting power of an Extremely Large Telescope (ELT) together with a high spatial resolution brought by adaptive optics (AO). Moreover, the galaxies need to be studied through a statistical approach, that require to be able to observe a wealth of them. Dealing with minimum integration times of the order 8 hours for each, the only way to achieve this is thus to multiplex the observations.

These requirements led in 2004 [1] to the concept of Multi-Object Adaptive Optics, or MOAO, fully inherited from the FALCON concept presented in 2001 [2]. A MOAO instrument is composed of individual systems that split the field. Some compensate the wavefront onto the small (few arcsecond) areas of the galaxies. They are driven by the wavefront information collected from the others systems : the off-axis wavefront sensors (WFS) that pick their signals from either natural or artificial stars across the field. The way the wavefront measurement are combined together in order to extract the wavefront control is the tomographic reconstruction.

EAGLE [3] is an MOAO instrument under conceptual study for the European Extremely Large Telescope (E-ELT). It will cover a field of view of 5 arcminutes. The top level requirements of the instrument call for 20 spectroscopic channels in the near infrared. Each of them will use 1 Deformable Mirror (DM) working in open-loop driven by the tomographic reconstruction using the wavefront information provided by the 6 Laser Guide Stars (LGS) WFS and 5 Natural Guide Stars (NGS) off-axis WFS. CANARY [4], the MOAO pathfinder for EAGLE, is an open loop and tomography experiment that will be installed in 2010 at the William Herschel Telescope $(D=4.2 \mathrm{~m})$ at La Palma, in the Canaries islands.

\section{Tomography}

One of the main adaptive optics limitation is the anisoplanetism angle. A guide star is required to sense the wavefront. The angular distance between the object and the guide star needs to be sufficiently short or the AO performance will decrease as the guide star gets further. This limiting angle is called

\footnotetext{
a e-mail: fabrice.vidal@obspm.fr
} the original work is properly cited. 
anisoplanetism angle. Because of this problem, the sky coverage is usually low whatever the size of the telescope. To solve the sky coverage problem, tomography is a method that uses the stars surrounding the target object even beyond the anisoplanetism angle to sense the wavefront. These stars may be either NGS or LGS. Although they are in off-axis directions they give lots of turbulence information. Tomography's goal is to predict the turbulence on the on-axis direction (i.e where the target object is) using the information from the off-axis WFS.

Severals tomography algorithms are currently under test in various laboratories. Ammons et al. [5] use the Spherical Waves algorithm in the MOAO case for a $10 \mathrm{~m}$ telescope, Costille et al. [6], Petit et al. [7] uses the Kalman based approach in Multi-Conjugate Adaptive Optics (MCAO) scheme, but none of them have been demonstrated in a real on-sky instrument.

Our concern is to develop a simple tomography algorithm that takes into account both the specificities of the atmosphere with the geometry of the tomographic problem, but also the real physical and opticals characteristics of the instrument.

\subsection{Tomography with covariance of slopes}

MOAO implies necessarily an open-loop operation of the DM, which means that we cannot measure directly the relation between each off-axis WFS and DM. In order to calibrate this relationship we propose to add another wavefront sensor, on axis, after the DM. The setup is equivalent to a classical closed loop AO scheme : this provides a way to calibrate a classical interaction matrix between the on-axis sensor and the DM. Of course during the real observation run, this on-axis sensor will not use the flux from the galaxy : it is only used for calibration purposes. This calibration matrix will be used together with a tomographic algorithm that is able to predict the on-axis WFS slopes.

In order to reconstruct the turbulence at a given direction we start from the real measurements of the instrument and try to use these data to learn the adequate reconstructor at a given time.

We propose to find a matrix, C that, transforms, at a given time $t$, the slopes measurements $\left(\vec{m}_{1}(t)\right.$, $\left.\vec{m}_{2}(t), \ldots, \vec{m}_{n}(t)\right)$ of all the off-axis WFS to the on-axis WFS slopes $\left(\vec{m}_{\text {onaxis }}(t)\right)$. With $\vec{m}_{\text {offaxis }}(t)$, the concatenation of the off-axis WFSs measurements, we can write the relation:

$$
\vec{m}_{\text {onaxis }}(t)=C \cdot \vec{m}_{\text {offaxis }}(t)
$$

The idea is to record lot of independent turbulence slopes measurements (ideally an infinity), seen by on and off-axis WFSs. We write $M_{\text {onaxis }}$ and $M_{\text {offaxis }}$ the set of these measurements and we have:

$$
M_{\text {onaxis }}=C \cdot M_{o f f a x i s}
$$

Because the matrices $M_{\text {onaxis }}$ and $M_{\text {offaxis }}$ are of rectangular shape, they cannot be inverted directly. We solve the matrix $\mathrm{C}$ by minimizing the quantity $\epsilon^{2}$ :

$$
\epsilon^{2}=\left\|C M_{\text {offaxis }}-M_{\text {onaxis }}\right\|^{2}
$$

Providing that the matrix $\left(M_{\text {offaxis }} M_{\text {offaxis }}{ }^{t}\right)$ can be inverted, the matrix $\mathrm{C}$ can be written as a Minimum Meam Square Estimator (MMSE) solution type (Fusco et al.[8]) and leads to:

$$
C=\left(M_{\text {central }} M_{\text {offaxis }}{ }^{t}\right)\left(M_{\text {offaxis }} M_{\text {offaxis }}{ }^{t}\right)^{-1}
$$

If we now consider that the matrix $M_{\text {offaxis }}$ is composed of an infinite set of turbulence slopes from all the off-axis WFS, then the matrix $\left(M_{\text {offaxis }} M_{\text {offaxis }}{ }^{t}\right)$ becomes the covariance matrix between the off-axis sensors slopes and for sake of clarity now called $C_{a a} . M_{\text {onaxis }}$ being an infinite set of vectors $\vec{m}_{\text {onaxis }}(t)$, the matrix $\left(M_{\text {central }} M_{\text {offaxis }}{ }^{t}\right)$ becomes the covariance matrix between the off-axis sensors slopes and the on-axis sensor slopes (called $C_{m a a}$ ).

If the wavefront perturbation is in the pupil plane, then $\mathrm{C}$ can be seen as a change of basis matrix from the off-axis sensors slopes to the on-axis one. By showing a common perturbation in the pupil plane, produced for instance by the M4 mirror of the E-ELT, we can calibrate the instrumental pupils 
mis-registrations between all the WFSs that may suffer of several parameters such as shifts ( $\mathrm{x}$ and $\mathrm{y}$ ), rotations $(\theta)$, magnification $(\mathrm{G})$ and eventually distorsion.

In the case the perturbation is not common in the pupil plane then, the matrix $\mathrm{C}$ behaves as a tomographic reconstructor in the on-axis measurement basis. It can be computed using all the WFS measurements, without any model at all, and can be written in the compact form:

$$
C=C_{m a a} \times C_{a a}^{-1}
$$

Unlike others tomographic algorithms, this reconstructor is only able to predict the slopes (and not the phase) that a on-axis sensor should see. The computation of the voltage to apply to the DM is given using the interaction matrix between the on-axis WFS and the DM.

\subsection{Examples of tomography procedures in open loop}

\subsubsection{The Learn and Apply algorithm}

The solution of the tomographic problem is given by equation 5 but still has to solve 2 main points to be implemented in a real system.

According to the equation 4, the 2 covariances matrices $C_{m a a}$ and $C_{a a}$ are needed to compute the tomographic reconstructor. Off-axis slopes are always provided so that we can compute, at any time, the $C_{a a}$ matrix easily. However, during a real observation, the on-axis slopes, provided by a central WFS, are needed to compute the $C_{\text {maa }}$ matrix. We can only compute half a tomographic reconstructor.

The second point is that, assuming that the on-axis slopes are provided, the tomographic reconstructor can be computed from the WFS measurements but only when an infinite time sequence from both on-axis and off-axis WFSs is provided. Of course, in the real world, we are limited by the statistical convergence of the covariance matrices.

To overcome these 2 problems we introduced a Kolmogorov (or eventually Von Karman) atmospheric model a priori. We wrote the theoretical expression of the covariance of slopes to fit the on-sky measured covariance matrices with several parameters from:

1. the turbulence strenght: for each layer $r_{0}$ and the outer scale $L_{0}$.

2. the geometric configuration: altitude $\mathrm{h}$ for each layer and pointing directions for each WFS.

3. and eventually, calibration parameters: $x, y, z, \theta$ and $G$

We have used a classical Levenberg-Marquardt fitting algorithm to retrieve the parameters from the raw measured data (the $C_{a a}$ matrix only or eventually both $C_{a a}$ and $C_{\text {maa }}$ matrices) and their theoretical expressions. This method allows us to learn the parameters (turbulence profile, geometric configuration and calibration coefficients) with only few seconds of recorded data. We use them to compute the real shape of the covariance $C_{a a}$ matrix which solves the statistical convergence problem.

The $C_{\text {maa }}$ matrix behaving as a projector, we can compute the real shape of this matrix by using the deduced turbulence parameters from the raw $C_{a a}$ matrix and adding the target position parameter. This way, we do not need the on-axis data to compute the other half part of the tomographic reconstructor C. The figures 1 and 2 shows an example of raw and fitted covariance matrices.

This new procedure allows us to measure all the parameters that need to be taken into account in a tomographic problem. The turbulence profile, geometric and calibration parameters are outputs of our algorithm. We called it the Learn and Apply (L\&A) algorithm. The L\&A procedure has the advantage to measure, on-sky, the optimal reconstructor without using any external a priori from the turbulence profile. It transforms the instrument as a huge SLODAR. It also takes into account the instrumental deviations because directly measured from the open-loop WFS measurements to fit exactly the instrument behaviour. 


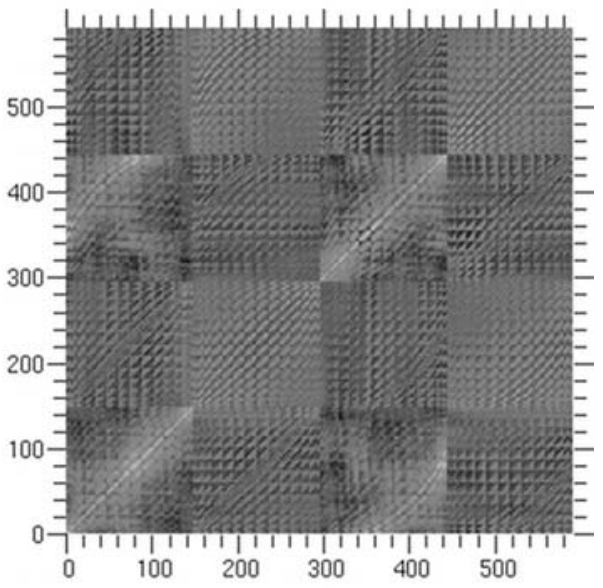

Fig. 1. Raw $C_{a a}$ covariance of slopes matrix between 2 off-axis $S H$.

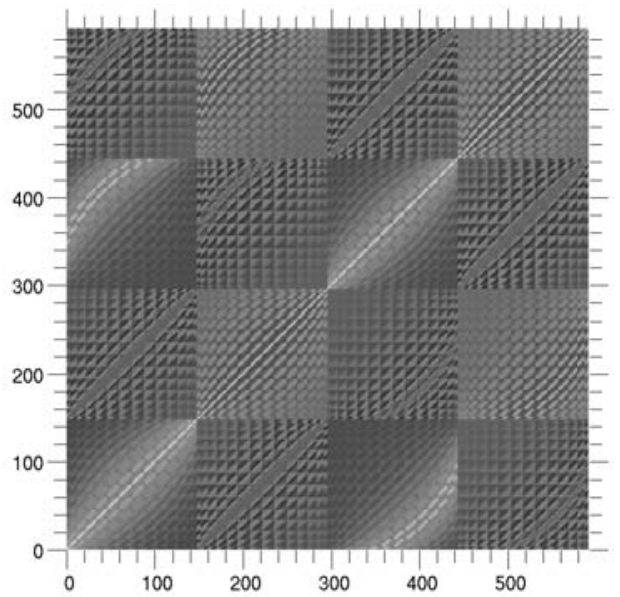

Fig. 2. Fitted $C_{a a}$ covariance of slopes matrix between 2 off-axis $\mathrm{SH}$.

\subsubsection{Using covariance matrices in a classical scheme: Apply only}

Previous section presented the principle of the Learn and Apply algorithm. Although the advantage of the L\&A method is to free from an external turbulence profiler (and consequently autonomous), we can still use any external $a$ priori to compute the 2 covariances matrices $C_{a a}$ and $C_{m a a}$, and finally the tomographic reconstructor C. The external profile given by a SLODAR or any $C_{n}^{2}(h)$ profiler can be used as an input to our slopes covariance matrices to compute the tomographic reconstructor.

In this case the algorithm "applies only" the given parameters. It is closer to a classical approach: the turbulence profile measurement is deduced thanks to an external system (SLODAR...) and is used as an input to a model in charge to the on-axis prediction.

\subsubsection{Retrieving turbulence profile from instrument data: Learn only}

In this strategy, we use the open-loop slopes to retrieve, in particular, the turbulence profile. These output parameters may be used for an other tomography algorithm. We transform the instrument into a SLODAR.

\section{Experimental results: MOAO with L\&A on SESAME bench}

SESAME is a multi-purpose bench for research and development in adaptive optics. We use it to simulate different multi-wavefront sensing AO concepts like MCAO and MOAO for the futures ELT's.

The goal of the experiment is to simulate the CANARY instrument with a MOAO correction using the Learn \& Apply scenario described in section 2.2.1. We also want to compare the correction quality between the SCAO and MOAO schemes to validate experimentally the L\&A algorithm.

\subsection{Experimental set-up}

We simulate a $4 \mathrm{~m}$ telescope and use a Kolmogorov phase screen plate to simulate a single turbulent layer. We work at a visible wavelength of $\lambda=630 \mathrm{~nm}$. At this wavelength we have $D / r_{0}=12.8$. The image we will get is equivalent to a J-Band Correction with a 1" seeing in V Band. We measure the off-axis and on-axis turbulence using $4(3+1)$ SH WFS with $14 \times 14$ subapertures. 
The layer is placed at an altitude of $9000 \mathrm{~m}$ and the asterism radius is 30 ". The pupil shift between the off-axis WFS and the on-axis WFS in this configuration is $31 \%$. The 3 off-axis WFS work in open-loop and provide the L\&A algorithm in charge to predict the on-axis wavefront. The correction is made using the $8 \times 8$ actuators of CANARY's deformable mirror controlled in open-loop. The DM is a piezostack type mirror and we characterized its typical open-loop error at 50nm rms. The equivalent fitting error is $85 \mathrm{~nm}$ rms for median conditions (1" seeing) in V Band. The wavefront correction is checked thanks to the on-axis sensor placed after the DM (also called the truth sensor). The truth sensor can also be used to close the loop in a classical SCAO scheme to compare the performance between the MOAO and SCAO scheme. An imaging camera records the on-axis Point Spread Function (PSF). Noise measurement at the SESAME bench is typically smaller than $10 \mathrm{~nm}$ rms.

We simulate a sampling frequency of $300 \mathrm{~Hz}$ with a wind speed of $10 \mathrm{~m} . \mathrm{s}^{-1}$. We recorded 1000 individual frames taken by the imaging camera and summed numerically them to perform a 3.33 seconds long exposure.

\subsection{Experimental results}

The uncorrected turbulence wavefront error (WFE) on 1000 frames is $650 \mathrm{~nm}$ rms. The uncorrected long exposure PSF, equivalent to a 3.33 seconds exposure is shown in figure 3 (left) and has a $1 \%$ SR and actually represents the seeing pattern.

In second we performed a classical SCAO correction using the on-axis (truth) sensor. The residual wave-front error after the SCAO correction is $100 \mathrm{~nm} \mathrm{rms}$. This value includes the fitting error, the calibrations errors (in close loop scheme). The long exposure PSF in SCAO is shown in figure 3 (center) and the SR is $39 \%$.

In third, we performed a full MOAO correction using the L\&A scenario (see section 2.2.1). The experiment is splitted in 2 steps. The first step is to record 1000 open-loop slopes to retrieve and compute the tomographic reconstructor. The second step is to test the learned reconstructor on the next 1000 draw numbers. We can compare the on-axis wavefront prediction provided by the L\&A algorithm and the real wavefront to correct measured by the truth sensor before the DM correction. The mean tomographic wfe on the 1000 tested draw number is $60 \mathrm{~nm}$ rms. The mean residual wavefront error after the DM open-loop correction is $110 \mathrm{~nm}$ rms. This value includes all the typical MOAO error budget (fitting error, tomography error, open-loop correction error, open-loop wave-front sensing error, aliasing...) except from the temporal error, not simulated in the experiment (no frame delay between sensing and correction). The long exposure PSF in MOAO scheme leads to SR=35\% and presented in figure 3 (right).
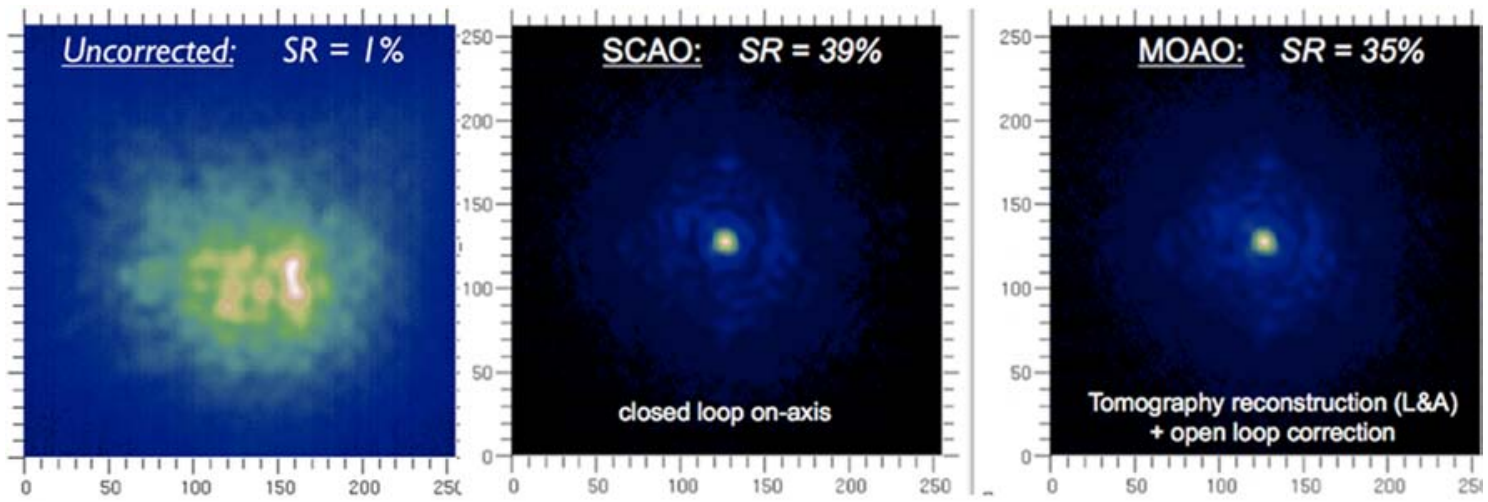

Fig. 3. 3.33 seconds equivalent exposure PSF. Left: Uncorrected turbulence. Center: SCAO correction Right: MOAO correction with 3 off-axis open-loop WFS at 30" from the target direction. 
The close results between the MOAO and SCAO schemes validates experimentally the tomography reconstruction using on-axis slopes instead of the phase. It also validates the use of the interaction matrix between the DM and the WFS in the on-axis target direction. This calibration scheme is a wellknown process and the measured interaction matrix between on-axis WFS and the DM is used in the L\&A procedure to link the predicted on-axis slopes and the voltage to apply to the mirror.

\section{Conclusion}

We have presented a new method to measure the tomographic reconstructor directly from the on-sky measurement with an MOAO instrument, while acquiring scientific data. The method, called Learn and Apply is based on the use of a supplementary on-axis wavefront sensor, placed downstream the DM, that is used to explain the measurements of all the WFSs in a common basis . This common basis is related to the DM in an unambiguous way thanks to the interaction matrix done on the on-axis sensor i.e. in the classical "old way" close-loop scheme.

Input data of the L\&A algorithm are only the sensors slopes while the output is the optimized tomographic reconstructor at a precise turbulence profile. This latter is also deduced from the direct measurements as well as the turbulence profile, positions of the sensors pupils versus the altitude, and eventually calibration parameters. Thanks to these retrieved parameters, we can compute a tomographic reconstructor without any external a priori from the turbulence profile and allows not to use the on-axis sensor during the real scientific observation. The tomographic reconstructor computed by the L\&A algorithm is able to compute the slopes that a on-axis sensor should see. The final computation of the voltage to apply to the DM is given using the interaction matrix between the on-axis WFS and the DM.

We voluntarily tried to reduce as far as possible the use of theoretical model and we are aware that this method is sub-optimal. However we think it may be better than using a model of the system (including DM, WFS, optical relations, etc) that could be unsuitable because of instrumental approximations, particularly in open loop. Moreover, our method allows, to some extent, to relax specifications for the tolerancing of an MOAO system because we measure the tomographic reconstructor as really "seen" by the instrument. The L\&A algorithm will be tested on-sky on the MOAO pathfinder, CANARY.

\section{References}

1. Dekany, Richard G.; Britton, Matthew C.; Gavel, Don T.; Ellerbroek, Brent L.; Herriot, Glen; Max, Claire E.; Veran, Jean-Pierre, "Adaptive optics requirements definition for TMT" SPIE Proc. 5490, (2004)

2. Hammer et al., "The FALCON Concept: Multi-Object Spectroscopy Combined with MCAO in Near-IR", Proceedings of the ESO Workshop Held in Garching, Germany, 11-15 Juni 2001, ESO ASTROPHYSICS SYMPOSIA. ISBN 3-540-43755-X. Edited by J. Bergeron and G. Monnet. Springer-Verlag, (2002) p. 139

3. Cuby J.G. et al., "EAGLE: an MOAO fed multi-IFU in the NIR on the E-ELT" SPIE Proc. volume 7014 (2008), pp. 70141K-70141K-11

4. Myers R.M. et al., "CANARY: the on-sky NGS/LGS MOAO demonstrator for EAGLE" SPIE Proc., Volume 7015 (2008) pp. 70150E-70150E-9

5. Ammons S. M. et al., "Integrated Laboratory Demonstrations of Multi-Object Adaptive Optics on a Simulated 10-Meter Telescope at Visible Wavelengths", submitted to PASP (2009)

6. Costille et al., "Optimization of MCAO performances: experimental results on ONERA laboratory MCAO bench" Proceedings of the SPIE, Volume 7015 (2008), pp. 701547-701547-10

7. Petit C. et al. , "First laboratory demonstration of closed-loop Kalman based optimal control for vibration filtering and simplified MCAO”, SPIE Proc. 6272 (2006)

8. Fusco T. et al., "Efficient phase estimation for large field of view adaptive optics", Optics Letters 1472 (1999), p 24 